\title{
A REMARK ON THE TIME CONSTANT IN FIRST-PASSAGE PERCOLATION
}

\author{
YVES DERRIENNIC, * Université de Bretagne Occidentale
}

\begin{abstract}
It is possible to deduce some information about the time constant $\mu$ without using Wierman and Reh's (1978) renewal theorem.
\end{abstract}

In the theory of first-passage percolation on the square lattice it is commonly deplored that Kingman's subadditive ergodic theorem provides no method for evaluating the time constant $\mu$. This letter aims to show that, at least in some cases, it is possible to deduce some information about this number $\mu$ from Kingman's arguments. We shall give a direct proof that $\mu(U)=0$ if the atom at $0 U(0)$ of the travel-time distribution satisfies $U(0) \geqq \frac{1}{2}$, without the renewal theorem that Wierman and Reh (1978) used in their original proof of this fact. (The converse implication, which is much harder, was proved by Kesten (1980).) Furthermore our method suggests an interesting question.

The setting of first-passage percolation theory is described by Smythe and Wierman (1978) and also in the recent survey of Wierman (1982). We follow the notations of this survey. We take $U$ to be the common distribution function of the independent random variables which represent the travel times of the bonds of the square lattice. We assume $U\left(0^{-}\right)=0$ and $\int_{0}^{+\infty} x U(d x)<+\infty$. The unrestricted point-to-point first-passage time from the point $(m, 0)$ to $(n, 0)$, denoted by $a_{m, n}$, is the minimum of the sum of the travel times of the bonds forming a self-avoiding path from $(m, 0)$ to $(n, 0)$, the minimum being taken over all such paths. From Kingman's theorem applied to the subadditive, stationary process $a_{m, n}$, it is known that $n^{-1} a_{0, n}$ converges almost surely and in mean to some constant. This constant is attached to the distribution $U$ and called the time constant' $\mu(U)$.

But Kingman's theory says more. According to the proof of the decomposition theorem of a subadditive process, the sequence $n^{-1} \sum_{r=0}^{n-1}\left(a_{0, r}-a_{1, r}\right)$ has a weak limit point in $L^{1}$, say $f$, and $\mu(U)=E(f)$. Now, under the assumption $U(0) \geqq \frac{1}{2}$, Harris's pioneering result tells us that any bounded set can be surrounded, almost surely, by an instantaneous closed circuit. By instantaneous we mean that the total travel time is 0 . Then it is obvious that, for $n$ large enough, $a_{0, n}-a_{1, n}$ equals the time to travel from $(0,0)$ to the smallest instantaneous closed circuit surrounding the two points $(0,0)$ and $(1,0)$, minus the time to travel from $(1,0)$ to the same circuit. Hence the sequence $a_{0, n}-a_{1, n}$ converges almost surely to the only possible weak limit point $f$ which is therefore integrable. Since the symmetry about the line $x=\frac{1}{2}$ leaves the probability law

Received 23 December 1982.

* Postal address: Faculté des Sciences et Techniques, Département de Mathematiques et Informatique, 6, Avenue Victor le Gorgeu, 29283 Brest Cedex, France. 
invariant and changes the value of $a_{0, n}-a_{1, n}$ into its opposite, the probability distribution of $f$ is symmetric about 0 and we get the desired result $E(f)=\mu(U)=0$. Furthermore the convergence in distribution of $a_{0, n}-a_{0, n-1}$ is proved, because $a_{0, n}-a_{1, n}$ and $a_{0, n}-a_{0, n-1}$ have the same distribution.

The method, which we have already used in some other instances (Derriennic (1980)), suggests the following question: in general is the sequence $a_{0, n}-a_{1, n}$ convergent?

\section{References}

DERRIENNIC, Y. (1980) Quelques applications du théorème ergodique sousadditif. Astérisque 74, 183-201.

Kesten, H. (1980) On the time constant and path length of first passage percolation. Adv. Appl. Prob. 12, 848-863.

Smythe, R. T. And Wierman, J. C. (1978) First-Passage Percolation on the Square Lattice. Lecture Notes in Mathematics 671, Springer-Verlag, Berlin.

Wierman, J. C. (1982) Percolation theory. Ann. Prob. 10, 509-524.

Wierman, J. C. AND ReH, W. (1978) On conjectures in first passage percolation theory. Ann. Prob. 6, 388-397. 